\title{
Empirische Darstellung des Versicherungsoutputs in Versicherungsgarantieeinheiten*
}

\author{
von Obi Mordi** und Franz Thole ${ }^{* * *}$
}

\section{Problemstellung}

Der Output von Versicherungsunternehmen wird gewöhnlich in monetären Grössen dargestellt, besonders mit den Prämieneinnahmen oder Versicherungssummen. Grund dafür ist der fehlende Massstab, mit dem nichtmonetäre Einheiten von Versicherungsoutput gemessen werden könnten.

Es liegt auf der Hand, dass eine blosse Addition von Geldbeträgen die differenzierten Risikoverhältnisse bei den einzelnen Versicherungen nicht zum Ausdruck bringen. Deshalb ist eine blosse Addition von Geldbeträgen unzweckmässig, weil das Versicherungsgeschäft das "Geschäft mit dem Risiko" (Karten, 1) darstellt. Es ist folglich nach einem Outputmass zu suchen, mit dem die Risikoverhältnisse bei der Versicherungsproduktion berücksichtigt werden.

Als Beitrag zur Lösung dieses Massproblems hat Mordi, (2) zunächst ein Produktkonzept für Versicherung vorgelegt und Produkteigenschaften ${ }^{1}$ beschrieben, die der Charakterisierung der Versicherungsproduktion dienen. In einem zweiten Beitrag (Mordi, 3) wurden die Messbarkeitseigenschaften des Produktkonzepts untersucht und die formale Messung des Outputs dargestellt. Danach kann der Versicherungsoutput in Versicherungsgarantie-Einheiten (VGE) gemessen werden.

Der vorliegende Beitrag soll die theoretischen Erkenntnisse am Beispiel der Kraftfahrtversicherung empirisch nachvollziehen und damit auf die Anwendbarkeit des Verfahrens in der Praxis hinweisen.

* Die Arbeit ist mit Unterstützung von Herrn Dr. J.J. Jappen, Vorsitzender des Vorstands der Öfentlichen Versicherung, Oldenburg, zustande gekommen, der die Verwendung von Daten des Versicherungsbestandes ermöglichte. Weiter hat Herr. Dipl.-Math. R. Willers, Oldenburg, die Arbeit angeregt und gefördert.

** Associate Professor für Versicherungswissenschaft an der Anambra State University of Technology in Enugu/Nigeria.

*** Dipl.-Math., Abteilungsdirektor der Offentlichen Versicherung Oldenburg.

${ }^{1}$ Immaterialität, Operationalität, Kommerzialität. 


\section{Output in der Kraftfahrversicherung : zwei empirische Fälle}

\subsection{Methodischer Ansatz}

Als Beispiele für die Outputmessung wurden die Fahrzeugteilversicherung und die Kraftfahrt-Haftpflichtversicherung verwendet. Da es sich um unbegrenzte Interessenversicherung bzw. um Erstrisikoversicherungen handelt, sind aus dem allgemeinen Messmodell ${ }^{2}$ die Summeneinheiten entbehrlich; denn in der Fahrzeugteilversicherung gibt es keine Versicherungssumme, in der Kraftfahrt-Haftpflichtversicherung nur eine Versicherungssumme im Sinne einer Höchstgrenze für Versicherungsleistungen (Deckungssumme).

Die Formel der Outputmessung ohne Summeneinheiten lautet:

$$
\text { Output }=\text { VGE }=\sum_{i=1}^{n} \frac{P_{i}}{P_{1}} \cdot J E_{i}
$$

wobei VGE Versicherungsgarantie-Einheiten, $P$ Bruttoprämie, $J E$ Jahreseinheiten bedeuten, und $i=1,2, \ldots, n$. Bei der Möglichkeit einer Aggregation von Teilbeständen lässt sich die Outputmessung mit dem Ansatz entsprechender Durchschnittswerte vereinfachen:

$$
\text { Output }=\text { VGE }=\sum_{j=1}^{m} \frac{\check{P}_{j}}{P_{l j}} \cdot J E_{j}
$$

wobei $\bar{P}_{j}$ die Durchschnittsprämien in der $j$. Teilsparte $(j=1,2, \ldots, m)$ darstellen (diese Teilsparten können wiederum verschiedene Klassen aufweisen). $P_{1 j}$ ist die niedrigste Prämie; $J E_{j}$ bedeutet die Jahreseinheiten in der $j$. Teilsparte.

Die Untersuchung der Kraftfahrtversicherung hat den Vorteil, dass die Gliederung der Statistiken im Rahmen des HUK-Verbandes in allen Versicherungsunternehmen ohne grossen Arbeitsaufwand vorgenommen werden kann.

\subsection{Fahrzeugteilversicherung}

Im ersten Beispiel wurde der Bestand an Fahrzeugteilversicherungen eines Versicherers zugrunde gelegt. Der Übersichtlichkeit halber ist die Rechnung auf die Versicherung von Personenwagen beschränkt worden (Wagniskennziffer 112 des HUK-Verbandsstatistik). Die Tarifierung von Fahrzeugteilversicherungen für Pkw wird nach Typklassen vorgenommen.

${ }^{2}$ Das allgemeine Modell lautet:

$$
\text { Output }=\text { VGE }=\quad \sum_{i=1}^{n} \frac{P S_{i}}{P S_{1}} \cdot S E_{i} \cdot J E_{i},
$$

wobei $P S=$ Prämiensatz, $S E=$ Summeneinheiten, $J E=$ Jahreseinheiten. Ohne Summeneinheiten, d.h. bei $S E=1$ und bei Ersatz von $P S$ durch $P$, entsteht Gleichung (1). 
Tabelle 1: Fahrzeugteilversicherung PKW (WKZ 112) 1982

(1)

\begin{tabular}{|c|c|c|c|c|}
\hline Typklasse & JE & J-Beitrag & Gesamt-Beitrag & Garantieeinheiten \\
\hline 10 & $1.286,90$ & 37,0 & $47.615,3$ & $1.286,90$ \\
\hline 11 & $7.077,30$ & 41,0 & $290.169,3$ & $7.842,4$ \\
\hline 12 & $5.724,5$ & 47,0 & $269.051,5$ & $7.271,7$ \\
\hline 13 & $9.104,40$ & 51,0 & $464.324,4$ & $12.549,3$ \\
\hline 14 & $6.686,8$ & 54,0 & $361.087,2$ & $9.759,1$ \\
\hline 15 & $4.272,60$ & 60,0 & $256.356,0$ & $6.928,5$ \\
\hline 16 & $4.219,4$ & 65,0 & $274.261,0$ & $7.412,5$ \\
\hline 17 & $2.652,40$ & 71,0 & $188.320,4$ & $5.089,7$ \\
\hline 18 & $1.839,10$ & 77,0 & $141.610,7$ & $3.827,3$ \\
\hline 19 & $2.570,5$ & 82,0 & $210.781,0$ & $5.696,8$ \\
\hline 20 & $2.821,5$ & 85,0 & $239.827,5$ & $6.481,80$ \\
\hline 21 & $2.232,70$ & 90,0 & $200.943,0$ & $5.430,9$ \\
\hline 22 & 580,60 & 97,0 & $49.334,2$ & $1.333,4$ \\
\hline 23 & 346,90 & 101,0 & $35.036,9$ & 946,9 \\
\hline 24 & 43,30 & 105,0 & $46.546,5$ & $1.258,0$ \\
\hline 25 & 55,10 & 110,0 & $6.061,0$ & 163,0 \\
\hline 26 & 366,3 & 118,0 & $43.223,4$ & $1.168,2$ \\
\hline 27 & 404,8 & 120,0 & $48.776,0$ & $1.312,9$ \\
\hline 28 & 346,2 & 127,0 & $43.967,4$ & $1.188,3$ \\
\hline 29 & 159,3 & 133,0 & $21.186,9$ & 572,6 \\
\hline 30 & 65,40 & 135,0 & $8.829,0$ & 238,6 \\
\hline 31 & 159,60 & 142,0 & $22.663,2$ & 612,5 \\
\hline 32 & 549,50 & 158,0 & $86.821,0$ & $2.346,5$ \\
\hline 33 & 530,40 & 186,0 & $98.654,4$ & $2.666,3$ \\
\hline 34 & 101,90 & 209,0 & $21.297,1$ & 575,6 \\
\hline 35 & 291,10 & 234,0 & $68.117,4$ & $1.841,0$ \\
\hline 36 & 51,90 & 251,0 & $13.026,9$ & 952,1 \\
\hline 37 & 146,8 & 297,0 & $43.599,6$ & $1.178,4$ \\
\hline 38 & 23,90 & 334,0 & $7.982,6$ & 215,7 \\
\hline 39 & 48,9 & 417,0 & $20.391,3$ & 551,1 \\
\hline 40 & 62,70 & 556,0 & $34.861,2$ & 942,2 \\
\hline & 55.150 .70 & & $3.664 .523,3$ & $99.040,2$ \\
\hline
\end{tabular}

Als Garantieeinheit 1 wird der Versicherungsoutput in der Fahrzeugteilversicherung für Pkw für ein Jahr mit der niedrigsten Prämie festgelegt. Da die Prämien mit den Typklassennummern ansteigen, ist die Prämie für die unterste Typklasse 10 die niedrigste überhaupt. Die Garantieeinheit 1 kann somit als Versicherungsschutz in der Fahrzeugteilversicherung für einen Pkw der Typklasse 10 definiert werden.

Tabelle 1 zeigt die Ermittlung der Garantieeinheiten je Typklasse beim untersuchten Versicherer. Spalte 1 bezeichnet die Typklasse, Spalte 2 enthält die Jahreseinheiten, das ist die auf volle Jahre umgerechnete Anzahl von Risiken. Spalte 3 enthält den Versicherungsbeitrag ohne Versicherungsteuer für eine Jahreseinheit. Spalte 4 ist das Beitragsvolumen 
Anlage Tabelle 2a: Berechnung durchschnittlicher Beitrag (100\%)

Tarifgruppe $\mathbf{R}$

$\begin{array}{cc}(1) & (2) \\ \text { Stärke } & \begin{array}{c}\text { Jahres } \\ \text { Einheiten }\end{array} \\ \text { Klasse } & 582,0 \\ 001 & 2.482,9 \\ 018 & 2.269,4 \\ 026 & 2.650,8 \\ 030 & 13.482,3 \\ 034 & 5.375,9 \\ 041 & 11.010,2 \\ 045 & 6.280,1 \\ 056 & 5.796,8 \\ 067 & 2.327,9 \\ 086 & 1.444,1 \\ 111 & 53.702,4\end{array}$

(3)

$100 \%$

Beitrag

318,0

443,0

526,0

570,0

674,0

727,0

802,0

871,0

910,0

$1.068,0$

$1.256,0$
(4)

(2) $\times(3)$

185.076

1.099 .925

1.193.704

1.510 .956

9.087.070

3.908.279

8.830 .180

5.469 .967

5.275 .088

2.486.197

$\frac{1.813 .790}{40.860 .232}$

$40.860 .232: 53.702,4=760,9$

Tarifgruppe A

(1)

Stärke
Klasse
001
018
026
030
034
041
045
056
067
086
111

$2.338 .133: 3.327,8=702,6$
(2)

Jahres

Einheiten

32,1

137,8

94,7

135,5

$1.046,8$

361,6

705,9

385,5

302,0

81,5

$\frac{44,4}{3.327,8}$
(3)

$100 \%$

Beitrag

294,0

413,0

495,0

532,0

634,0

684,0

752,0

820,0

861,0

$1.009,0$

$1.185,0$
(4)

(2) $\times(3)$

9.437

56.911

46.877

72.086

663.671

247.334

530.837

316.110

260.022

82.234

52.614

2.338 .133

Tarifgruppe B

(1)

Stärke

001

018

026

030

034

041

045

056

067

086

111
(2)

Jahres

Einheiten

241,9

$1.192,4$

$1.097,8$

$1.105,3$

$6.049,5$

$2.501,3$

$4.989,0$

$2.791,0$

$2.237,8$

587,8

$\frac{180,1}{22.973,9}$
(3)

$100 \%$

Beitrag

260,0

362,0

428,0

462,0

548,0

591,0

651,0

708,0

741,0

869,0

$1.023,0$
(4)

(2) $\times(3)$

62.894

431.649

469.858

510.649

3.315 .126

1.478.268

3.247.839

1.976.028

1.658.210

510.798

184.242

13.845 .561

$13.845 .561: 22.973,9=602,7$ 
Tabelle $2 b$ : Berechnung Beitragssatz

Tarifgruppe $\mathbf{R}$

(1) S/SFR

Klasse

$\mathrm{S} 3$

S 2

S 1

SF $1 / 2$

SF $1 / 2$

SF 2

SF 3

SF 4

SF 5

SF 6

SF 7

SF 8

SF 9-13

Tarifgruppe A

Tariffgruppe B

(1)

Slasse

$$
\text { S } 3
$$

S 2

S 1

SF $1 / 2$

SF 1

SF 2

SF 3

SF 4

SF 5

SF 6

SF 7

SF 8

SF 9-13

(1)
(2) Jahreseinheiten $\%$-Anteil

0,02

0,06

0,47

1,37

4,61

5,47

5,39

6,62

6,41

6,02

5,73

5,13

4,87

47,83

(2)

S/SFR

Klas 3

S2

S 1

0

SF 1

SF 2

SF 3

SF 4

SF 5

SF 6

SF 7

SF 8

SF 9-13
Jahreseinheiten $\%$-Anteil

0,03

0,01

0,43

0,03

4,37

4,28

4,00

4,12

3,43

3,00

3,30

2,84

2,79

67,37

(2)

Jahreseinheiten

\%-Anteil

0,01

0,05

0,42

0,71

4,21

4,99

5,05

5,71

5,46

5,32

5,85

5,30

4,88

52,04
(3)

Beitragssatz

$\%$

200

175

175

125

100

85

70

65

60

55

50

45

40

(3)

Beitragssatz

$\%$

200

200

175

175

125

100

85
70

65

60

55

50

45

40

(3)

Beitragssatz

$\%$

200

200

175

175

125

100

85

70

65

60

55

50

45

40
(4)

(2) $\times(3)$

4

12

240

576

547

458

464

417

361

315

257

219

1.913

5.865

(4)

(2) $\times(3)$

$\begin{array}{r}6 \\ 2 \\ 75 \\ 5 \\ 546 \\ 428 \\ 340 \\ 288 \\ 223 \\ 180 \\ 182 \\ 142 \\ 126 \\ 2.695 \\ \hline 5.238\end{array}$

(4)

(2) $\times(3)$

2

10

74

124

526

499

428

400

355

319

322

265

220

$\frac{2.082}{5.626}$ 
Tabelle 3: Kraftfahrzeughaftpflicht Pkw (WKZ 112) 1982

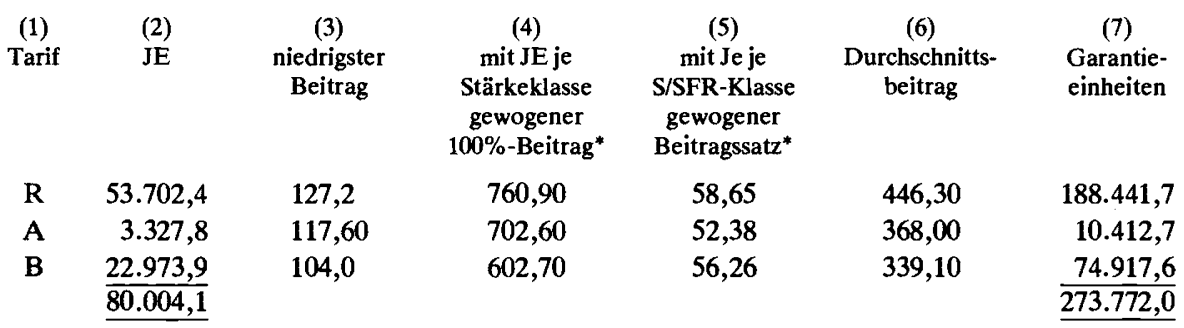

* Berechnung in Tabelle 2

der Typklasse (Spalte 2 mal Spalte 3). Spalte 5 gibt schliesslich die Garantieeinheiten je Typklasse an; in Typklasse 10 ergeben sie sich unmittelbar aus Spalte 2, bei allen anderen durch die Division von Spalte 4 durch Spalte 3 (oder Spalte 2 mal Spalte 3, dividiert durch Spalte 3 für Typklasse 10).

Folgendes ist das Ergebnis der Outputmessung: Entsprechend der Bestandszusammensetzung bedeuten 55 150,5 Jahreseiheiten 99 040,2 Garantieeinheiten. Dieses Ergebnis erhält man auch, wenn die Gesamtprämieneinnahme ( 3664523,3 ) durch den niedrigsten Beitrag (37) dividiert wird (Rundungsdifferenz). Man erhält auf diese Weise ein Mass für den Output an (Versicherungsgarantien), der die Anzahl der Verträge (Jahreseinheiten) und die Prämienhöhe für die bedingungsgemässen Versicherungen repräsentiert.

\subsection{Kraftfahrt-Haftpflichtversicherung}

Als zweites Beispiel wurde die Haftpflichtversicherung für Pkw geprüft. Die Prämien werden hier vor allem nach der Fahrzeugstärke, nach Beruf und Region differenziert. Weiter erfolgt eine Einteilung in Schadenfreiheits- und Schadenklassen. Zur Vereinfachung wurde die Regionalisierung der Prämie nicht weiter beachtet.

Für die Berechnung der Garantieeinheiten als Mass des Outputs aus dem Versicherungsbestand wird zunächst das Mass für die Garantieeinheit 1 festgesetzt. Für jede Berufsgruppe bedeutet der niedrigste Prämienbetrag die Garantieeinheit 1; bedingt durch die Tarifstruktur ist dies die Prämie für die unterste Stärkeklasse mit dem höchsten Schadenfreiheitsrabatt.

Danach wurde entsprechend den Beitragssätzen der Schadenfreiheits- und Schadenklassen sowie den in den Klassen jeweils befindlichen Jahreseinheiten ein durchschnittlicher Beitragssatz für diesen Bestand ermittelt. Durch Multiplikation mit dem (nach Jahreseinheiten) gewogenen durchschnittlichen $100 \%$-Beitrag ergibt sich der durchschnittliche gezahlte Beitrag; die Einzelrechnungen sind in Tabelle 2 dargestellt. Die Ergebnisse sind in Tabelle 3 festgehalten: Die 80 004,1 Jahreseinheiten bedeuten 273772 Garantieeinheiten, also etwas das Dreieinhalbfache.

Nach diesem Beispiel wurde ein Mass für die Outputs dargestellt, das die Anzahl der Risiken und dem Umfang des jeweils versicherten Risikos, ausgedrückt durch die Prämie, berücksichtigt. 


\section{Schlussbemerkungen}

Die beiden Beispiele aus der Kraftfahrtversicherung sollen zeigen, dass sich der Versicherungsoutput "messen" lässt, und zwar nicht in monetären Grössen. Die Berechnungsweise ist einfach. Im Interesse von Vergleichen zwischen einzelnen Versicherern sollte die Verbandsstatistik normiert werden. Dann lassen sich auch die Entwicklungen in der Zeit bei den einzelnen versicherten Risiken und Produktgruppen verfolgen. Weitere Bewertungen des Verfahrens sind bei Mordi (2) angegeben.

Die einzelnen Versicherungsunternehmen der Kraftfahrtversicherung kalkulieren die Prämien nach betriebsindividuellen Rechnungsgrundlagen. Das füuhrt - in Grenzen - zu Prämienunterschieden. Prämienunterschiede bei gegebenem Risiko bedeuten verschieden grosse Outputs. Weitere Bestimmungsgrössen für den Output in der Kraftfahrtversicherung sind die Anzahl der versicherten Risiken und die Zeitdauer der Versicherungs verträge.

\section{LITERATURHINWEIS}

Karten, Walter: Grundlagen einer versicherungspolitischen Risikopolitik, in: Geld und Versicherung, Festschrift Seuss (jung, M., Lucius, R.R., Seifert, W.G. Hrsg), Karlsruhe 1981, S. $135 \mathrm{ff}$.

Mordi, Obi : Das Produktkonzept der Versicherung: Eine Alternative Interpretation (erin : ZVersWiss, 1985, S. 81-93.

- derselbe - : Outputmessung in der Versicherungswirtschaft - Konzeptionelle Wirtschafts und versicherungstechnische Überlegungen (im gleichen Heft der Geneva Papers).

\section{Summary: Empirical Measurement of the Production of an Insurance Company}

In this article an empirical possibility to measure the output of an insurance company is shown, using as examples the third-party motor insurance and the vehicle insurance. The statistics of claims of the german motor insurance industry and the rates of a german motor insurance company serve as the basis for this measurement. 